\title{
Source degenerate identification problems with smoothing overdetermination
}

\author{
Fadi Awawdeh ${ }^{1,2^{*}}$, AK Alomari ${ }^{3}$ and Ibraheem Abu-Falahah'
}

${ }^{\text {*Correspondence: }}$

awawdeh@hu.edu.jo

1 Department of Mathematics,

Hashemite University, Zarqa, 13115,

Jordan

${ }^{2}$ Mathematics and Sciences

Department, Dhofar University,

Salalah, 211, Oman

Full list of author information is

available at the end of the article

\begin{abstract}
We consider degenerate identification problems with smoothing overdetermination in abstract spaces. We establish an identifiability result using a projection method and suitable hypotheses on the operators involved and develop an identification method by reformulating the problem into a nondegenerate problem. Then we use perturbation results for linear operators to solve the regular problem. The introduced identification method permits one to solve the problems under the minimum restrictions on the input data. Finally, we provide applications to degenerate differential equations that appear in mathematical physics to support the theoretical results.
\end{abstract}

\section{Introduction}

In certain systems in physics and engineering, there arise differential equations with degeneracy. The system with a noninvertible operator at the derivative is considered as a good example of such systems. Most research in the literature was devoted to nondegenerate systems which do not cover fully the diversity of problems arising in theory and applications. In the case of degenerate problems, the analysis becomes more complicated, and somewhat different techniques are required. On the other hand, most of the important problems modeled by nonlinear degenerate partial differential equations are truly challenging and require further new approaches and techniques, and need our full attention and further efforts.

With this aim, this work is concerned mainly with an identification problem for a firstorder degenerate system. Let $X$ be a Banach space endowed with the norm $\|\cdot\|$, and let $M$ and $L$ be two closed linear operators in $X$. Let $z \in X$; let $\phi:[0, \tau] \rightarrow \mathbb{R}_{+}, \tau>0$, be a $\mathcal{C}^{1}$ functional, and let us consider the following identification problem:

$(\mathcal{I P} 1)$ Given $v_{0} \in X$ and $g \in \mathcal{C}^{1}([0, \tau] ; \mathbb{R})$, find $f \in \mathcal{C}([0, \tau] ; \mathbb{R})$ and a strict solution $v \in$ $\mathcal{C}^{1}([0, \tau] ; X)$ to the degenerate Cauchy problem

$$
\left\{\begin{array}{l}
\frac{d M v}{d t}=L v(t)+f(t) z, \quad 0 \leq t \leq \tau \\
M v(0)=M v_{0}
\end{array}\right.
$$

satisfying the additional condition

$$
\phi[M v(t)]=g(t), \quad 0 \leq t \leq \tau .
$$

(c) The Author(s) 2017. This article is distributed under the terms of the Creative Commons Attribution 4.0 International License (http://creativecommons.org/licenses/by/4.0/), which permits unrestricted use, distribution, and reproduction in any medium, provided you give appropriate credit to the original author(s) and the source, provide a link to the Creative Commons license, and indicate if changes were made. 
More precisely, we are concerned with the determination of the conditions under which we can identify $f \in \mathcal{C}([0, \tau] ; \mathbb{R})$ such that $v$ is a strict solution to the above problem, i.e.,

$$
M v \in \mathcal{C}^{1}([0, \tau] ; X), \quad L v \in \mathcal{C}([0, \tau] ; X)
$$

It should be emphasized that our identification problem $(\mathcal{I P} 1)$ is related to applications in control theory. There are some ways to consider the $(\mathcal{I P} 1)$ as being naturally connected with an optimal control problem [1].

\subsection{Background and related work}

In many applications concerning identification problems for PDEs, the source term may be unknown. The best known applications of these types of problems occur when finding a pollution source intensity by measurements of the pollutant concentrations and for identifying the laser beam intensity and trajectory in the heat equation corresponding to a given-in-advance temperature distribution [2].

One difficulty in inverse source problems is the absence of identifiability (uniqueness) of an arbitrary source as was shown in [3]. Thus, a well-posed source identification problem can be obtained if some a priori information is known. This additional information may be described by certain conditions on the admissible sources induced by the physical problem. For example, measuring the temperature by a perfect sensor of finite size in the heat source identification problem. A space-dependent source identification problem is considered by Cannon in [4]. Farcas and Lesnic studied a source dependent only on time [5]. Separable sources are analyzed in detail by Engl et al. [6]. A moving source whose spatial support is contained within a ball with a given radius is treated by Kusiak and Weatherwax [7]. The sum of $m$ stationary sources with time-varying intensities are considered in [3].

It is worth noting that the identification problem, with linear source, related to nondegenerate systems is widely studied in the literature concerning inverse problems for PDEs. For more details on this subject, we refer to the monograph of Prilepko et al. [8, Chapter 7], the two papers by Orlovsky $[9,10]$ and the references given there.

The direct problem of (IPP1), i.e., $M=I$, was first considered by Lorenzi [11]. In this paper an explicit solution formula was established under the condition that the operator $L$ is bounded. As a matter of fact, more results were obtained by Prilepko et al. [8], where the operator $L$ was supposed to be a generator of a $c_{0}$-semigroup. In the work of Lorenzi [11] it was supposed with regard to problem $(\mathcal{I P} 1)$ that $f(t)$ is known, $z$ is unknown and the semigroup generated by $L$ is analytic, that is, the case of parabolic equation occurred. A direct nonlinear version of $(\mathcal{I P} 1)$ with $L$ being the infinitesimal generator of a compact $c_{0}$-semigroup of contractions was discussed by Lorenzi [1]. General results for secondorder identification differential problems were obtained by Awawdeh [12].

In contrast, very few results are known for degenerate inverse problems, even though this class of systems occurs in many interesting problems in engineering and applied mathematics. One of the first results on this subject was discussed in [13] where $\lambda=0$ is assumed to be a simple pole for the resolvent operator $\left(\lambda I+M L^{-1}\right)^{-1}$. Later, more theoretical results were obtained in [14], where the pair $(M, L)$ of closed linear operators is weakly parabolic, i.e.,

$$
\left\|M(\lambda M+L)^{-1}\right\|_{\mathcal{L}(X)} \leq C(1+|\lambda|)^{-\beta}, \quad \forall \lambda \in \Sigma_{\alpha}
$$


with

$$
\Sigma_{\alpha}=\left\{\lambda \in \mathbb{C}: \operatorname{Re} \lambda \geq-c(1+\operatorname{Im} \lambda)^{\alpha}\right\},
$$

where $C, c>0,0<\beta \leq \alpha \leq 1, \beta+\alpha>1$ and $\mathcal{L}(X)$ denotes the space of all bounded linear operators on $X$ with the uniform norm. Moreover, in [15] the representation of the space $X$ as a direct sum

$$
X=\mathcal{N}(A) \oplus X_{1}=\mathcal{R}(A) \oplus X_{2}
$$

where $M$ is assumed to have a closed kernel $\mathcal{N}(M)$ and a closed range $\mathcal{R}(M)$, and $X_{1}, X_{2}$ are closed subspaces of $X$, is essential to guarantee a unique strict solution. Second-order degenerate identification differential problems were studied in [16]. Awawdeh and Obeidat introduced the possibly multivalued linear operator $A=L M^{-1}$ following Favini and Yagi [17] and obtained some results concerning the solvability of the $(\mathcal{I P} 1)$ in [18]. Fedorov and Ivanova [19] studied an identification problem for a degenerate evolution equation with overdetermination on the solution semigroup kernel. Very recent results have been obtained in [20] for degenerate integro-differential equations.

It is important to mention that there are few studies about abstract degenerate fractional differential equations since they are essentially complicated and their theory is still in its early stages. The first paper on this subject was provided in Fedorov and Ivanova [21] for studying an identification problem for time-fractional order partial differential equations. After that, some works have been published. In [22], Kostić considered abstract degenerate fractional differential inclusions in Banach spaces and gave uniqueness results. We refer the reader to [23-25] for further information about abstract degenerate differential equations with integer order derivatives.

\subsection{Contributions}

This paper builds on the works of Al Horani [13-16] and Favini and Marinoschi [26]. The objective of these works was to obtain theoretical identifiability and local stability results for degenerate differential equations. That is, solution schemes that work well even in the case of systems with a noninvertible operator at the derivative. It is worth noting here that the classical theory of $c_{0}$-semigroups is not obviously applicable for problem (IP 1$)$ because $M^{-1}$ is not continuous in general. Another option is to use an operational method. These methods have been developed mainly for linear systems arising from elliptic and parabolic PDEs.

We propose an identification method based on reformulating the inverse problem $(\mathcal{I} \mathcal{P} 1)$ into an equivalent nondegenerate problem. As a first step, a projection method is used to reduce the problem to a regular abstract inverse problem. The problem is then handled with the help of some perturbation results for linear operators. Our smoother result is based on constructing an exact representation of the solution avoiding the calculations of some resolvent estimates of the involved operators and the study of some properties of the multivalued linear operator $L M^{-1}$.

At first we will solve the inverse problem $(\mathcal{I} P 2)$

$$
\frac{d}{d t} B w-w=f(t) z, \quad 0<t \leq T
$$




$$
\begin{aligned}
& B w(0)=w_{0}, \\
& \phi[B w(t)]=g(t), \quad 0<t \leq T,
\end{aligned}
$$

where the linear operator $B$ has $\lambda=0$ as a pole of $(\lambda-B)^{-1}$ of order $k+1$ and $f, g, z, \phi$ are as in $(\mathcal{I P} 1)$. We begin by constructing the fundamental solution to problem $(\mathcal{I P} 2)$ using a projection method and some perturbation results for linear operators. We believe that our results in Theorem 1 and Theorem 2, in which a fundamental solution with satisfactory properties is constructed for $(\mathcal{I P} 2)$, are of some independent interest. This fundamental solution enables us to solve not only $(\mathcal{I P} 2)$ but also the original problem $(\mathcal{I P} 1)$ by using the operator transformation $B=M L^{-1}$. In Theorem 3, we establish existence and uniqueness results for $(\mathcal{I P} 1)$ and give an explicit solution formula. It is remarkable that in the results in Theorem 3, no conditions on the resolvent estimates of the operators $M$ and $L$, or in the existence of bounded inverse of $M$, are needed.

We apply the abstract results for problems of mathematical physics. The method performs well with differential equations of the Sobolev type. Thus, despite the restrictive assumptions we impose in order to obtain the identifiability results, we are able to construct a scheme that is efficient for a broader set of degenerate identification problems.

\subsection{Organization of the paper}

The rest of the paper is organized as follows. In Section 2 the existence and uniqueness of the solution to the identification problems $(\mathcal{I P} 1)$ and $(\mathcal{I P} 2)$ are proved under suitable assumptions on the data. Furthermore, an implicit representation of the solution $(v, f)$ is obtained. An identification problem for a first-order differential equation of the Sobolev type, subjected to an overdetermination expressed by means of a Lebesgue integral, is presented in Section 3. The results so found are then applied to such a problem. Section 4 offers one possible way of applying the abstract results to problems of mathematical physics.

\section{Main results}

Let us first consider the following identification problem:

$(\mathcal{I P} 2)$ Given $w_{0} \in X, \phi:[0, \tau] \rightarrow \mathbb{R}_{+}, \tau>0$, a functional on $X$, and $g \in \mathcal{C}^{1}([0, \tau] ; \mathbb{R})$ determine the conditions under which we can identify $f \in \mathcal{C}([0, \tau] ; \mathbb{R})$ such that $w$ is a strict solution to the Cauchy problem

$$
\begin{aligned}
& \frac{d}{d t} B w-w=f(t) z, \quad 0<t \leq \tau, \\
& B w(0)=w_{0},
\end{aligned}
$$

satisfying the additional condition

$$
\phi[B w(t)]=g(t), \quad 0<t \leq \tau .
$$

Here $B$ is a closed linear operator in $X$ which has $\lambda=0$ as a pole of $(\lambda-B)^{-1}$ of order $k+1$. 
Throughout, for any linear operator $S$ in $X, \mathcal{R}(S)$ denotes the range of $S$ and $\mathcal{K}(S)$ is the null space of $S$. Recall that the decomposition

$$
X=\mathcal{K}\left(B^{m}\right) \oplus \mathcal{R}\left(B^{m}\right)
$$

holds for every $m \geq k+1$. Moreover, $\mathcal{R}\left(B^{m}\right)=\overline{\mathcal{R}\left(B^{m}\right)}=\mathcal{R}\left(B^{k+1}\right), \mathcal{K}\left(B^{m}\right)=\mathcal{K}\left(B^{k+1}\right)$ (see $[27$, p.229]). If $\Gamma:|\lambda|=\epsilon$ is a circumference of sufficiently small radius with $|\lambda| \leq \epsilon$ not containing singularities other than $\lambda=0$ and if

$$
P=\frac{1}{2 \pi i} \int_{\Gamma}(\lambda-B)^{-1} d \lambda
$$

then $P$ is a projection onto $\mathcal{K}\left(B^{k+1}\right)$ and $\mathcal{R}(I-P)=\mathcal{R}\left(B^{k+1}\right)$. Furthermore, it is easy to verify that

$$
\left\|\lambda^{k+1}(\lambda-B)^{-1}\right\|_{\mathcal{L}(X)} \leq C
$$

for any $\lambda$ such that $0<\lambda \leq \epsilon$.

Since $\mathcal{K}\left(B^{k}\right) \subset \mathcal{K}\left(B^{k+1}\right)$, we observe that, if $f \in \mathcal{C}([0, \tau] ; \mathbb{R})$, then the identification problem $(\mathcal{I P} 2)$ is equivalent to the couple of problems

$$
\begin{aligned}
& \frac{d}{d t} B_{1}(I-P) w-(I-P) w=f(t)(I-P) z, \\
& B_{1}(I-P) w(0)=(I-P) w_{0}, \\
& \phi\left[B_{1}(I-P) w(t)\right]=g(t),
\end{aligned}
$$

and

$$
\frac{d}{d t} B_{2} P w-P w=f(t) P z
$$

where $B_{1}$ and $B_{2}$ denote the parts of $B$ in $\mathcal{R}\left(B^{k+1}\right)$ and in $\mathcal{K}\left(B^{k+1}\right)$, respectively. Of course, problem (6)-(8) is a problem in the space $\mathcal{R}\left(B^{k+1}\right)$. It is also known by [28, p.178] that the spectra of $B_{1}$ and $B_{2}$ coincide with the spectrum of $B$ minus $\{0\}$ and with $\{0\}$, respectively. Hence, $B_{2} \in \mathcal{L}\left(\mathcal{K}\left(B^{k+1}\right)\right)$. On the other hand, $B_{1}$ is a closed operator in $\mathcal{R}\left(B^{k+1}\right)$, mapping $\mathcal{D}\left(B_{1}\right)=\mathcal{D}(B) \cap \mathcal{R}\left(B^{k+1}\right)$ onto $\mathcal{R}\left(B^{k+1}\right)$ in a one-to-one fashion.

Let $u=B_{1}(I-P) w$, then problem (6)-(8) becomes

$$
\begin{aligned}
& \frac{d u(t)}{d t}=B_{1}^{-1} u(t)+f(t)(I-P) z, \quad 0 \leq t \leq \tau, \\
& u(0)=u_{0} \\
& \phi[u(t)]=g(t), \quad 0 \leq t \leq \tau,
\end{aligned}
$$

where $u_{0}=(I-P) w_{0}$. It is seen in [27, Chapter VIII, Section 3] that $B_{1}$ is an abstract potential operator in $\mathcal{R}\left(B^{k+1}\right)$, i.e., $B_{1}^{-1}$ generates a $\mathrm{c}_{0}$-semigroup in $\mathcal{R}\left(B^{k+1}\right)$.

As the next stage, we are going to use a method coupled with perturbation theory for linear operators for the solvability of the inverse problem (10)-(12). 
Applying the linear functional $\phi$ to both sides of the differential equation (10) and using (12), we obtain the following equation for $f$ :

$$
g^{\prime}(t)-\phi\left[B_{1}^{-1} u(t)\right]=f(t) \phi[(I-P) z]
$$

If we assume that the following solvability condition is satisfied

$$
\gamma(z)^{-1}=\phi[(I-P) z] \neq 0,
$$

we can rewrite equation (13) in the form

$$
f(t)=\gamma(z)\left(g^{\prime}(t)-\phi\left[B_{1}^{-1} u(t)\right]\right)
$$

Substituting (15) in (10), we get

$$
u^{\prime}(t)=B_{1}^{-1} u(t)+\gamma(z)\left(g^{\prime}(t)-\phi\left[B_{1}^{-1} u(t)\right]\right)(I-P) z
$$

Clearly (16) implies

$$
u^{\prime}(t)-\left(B_{1}^{-1} u(t)+(-\gamma(z))\left(\phi\left[B_{1}^{-1} u(t)\right]\right)(I-P) z\right)=\gamma(z) g^{\prime}(t)(I-P) z .
$$

When $(X,\|\cdot\|)$ equipped with the graph norm

$$
\|x\|_{B_{1}^{-1}}=\|x\|+\left\|B_{1}^{-1} x\right\|,
$$

the domain $\mathcal{D}\left(B_{1}^{-1}\right)$ becomes a Banach space embedded continuously into the space $X$, which we shall denote by $X_{B_{1}^{-1}}$. In what follows we agree to consider the operator

$$
T x=-\gamma(z)\left(\phi\left[B_{1}^{-1} x\right]\right)(I-P) z .
$$

Then equality (17) becomes

$$
u^{\prime}(t)-\left(B_{1}^{-1}+T\right) u(t)=\gamma(z) g^{\prime}(t)(I-P) z
$$

The boundedness of the operator $T$ in $X_{B_{1}^{-1}}$ follows from the estimate

$$
\begin{aligned}
\|T\|_{B_{1}^{-1}} & \sup _{\|x\|_{B_{1}^{-1}}=1}\|T x\| \\
& =\sup _{\|x\|_{B_{1}^{-1}=1}}\left\|-\gamma(z)\left(\phi\left[B_{1}^{-1}(x)\right]\right)(I-P) z\right\| \\
& \leq \sup _{\|x\|_{B_{1}^{-1}=1}}|\gamma(z)|\|(I-P) z\|\|\phi\|\left\|B_{1}^{-1} x\right\| \\
& \leq|\gamma(z)|\|(I-P) z\|\|\phi\| .
\end{aligned}
$$

The following perturbation theorem will justify that the operator $B_{1}^{-1}+T$ is the generator of a $c_{0}$-semigroup $S(t), t \geq 0$, on $X$. 
Theorem 1 ([29]) Let $X$ be a Banach space, and let $A$ be the infinitesimal generator of a $c_{0}$-semigroup $T(t)$ on $X$. If $B: X_{A} \longrightarrow X_{A}$ is a continuous linear operator, then $A+B$ is the infinitesimal generator of a $c_{0}$-semigroup on $X$.

Since $u_{0} \in D\left(B_{1}^{-1}\right)$, it is well known that the Cauchy problem (10)-(11) has a unique solution

$$
u(t)=S(t) u_{0}+\gamma(z) \int_{0}^{t} S(t-s) g^{\prime}(s)(I-P) z d s
$$

Hence, by (15) and (20), $f(t)$ is uniquely determined. Therefore, the reduced problem (10)(12) possesses a unique solution $(u, f)$. It is also easy to verify that $(u, f)$ is a solution of (10)-(12) if and only if $(w, f)$ is a solution to (3)-(5).

Using (15) and (20) we have

$$
f(t)=\gamma(z)\left(g^{\prime}(t)-\phi\left[B_{1}^{-1} S(t) u_{0}\right]\right)-\gamma(z) \phi\left[\int_{0}^{t} B_{1}^{-1} S(t-s) g^{\prime}(s)(I-P) z d s\right] .
$$

As regards (9), we point out by [27, p.328] that

$$
\left(\lambda B_{2}+I\right)^{-1}=\sum_{i=0}^{\infty}(-1)^{i} \lambda^{i} B_{2}^{i}, \quad \lambda \neq 0
$$

and hence all the powers $B_{2}^{m}$ vanish provided that $m \geq k+1$. Since $f(t)$ is now known, then equation (9) possesses the unique solution

$$
P w(t)=\sum_{j=0}^{k} B_{2}^{j} P f^{(j)}(t) z, \quad 0 \leq t \leq \tau .
$$

We can verify that

$$
w(t)=\sum_{j=0}^{k} B^{j} f^{(j)}(t) z, \quad 0 \leq t \leq \tau,
$$

satisfies equation (3).

Now, we know that

$$
(I-P) w=B_{1}^{-1} u=B_{1}^{-1} S(t) u_{0}+\gamma(z) \int_{0}^{t} B_{1}^{-1} S(t-s) g^{\prime}(s)(I-P) z d s
$$

and so,

$$
\begin{aligned}
w(t) & =P w(t)+(I-P) w(t) \\
& =\sum_{j=0}^{k} B_{2}^{j} P f^{(j)}(t) z+B_{1}^{-1} S(t) u_{0}+\gamma(z) \int_{0}^{t} B_{1}^{-1} S(t-s) g^{\prime}(s)(I-P) z d s .
\end{aligned}
$$


We note that no initial condition can be assigned arbitrarily to $B w(t)$ at $t=0$. In fact, all admissible initial values $w_{0}$ must be of the form

$$
w_{0}=(I-P) w_{1}+\sum_{j=0}^{k-1} B^{j+1} P f^{(j)}(0) z
$$

where $w_{1} \in X,(I-P) w_{1} \in \mathcal{R}\left(B^{k+1}\right)$. If $B \in \mathcal{L}(X)$, taking $w_{1}=\left(B_{1}^{k}\right)^{-1} w_{0}$, for every $w_{0} \in$ $\mathcal{R}\left(B^{k+1}\right)$, there exists a solution $w$ to the identification problem $(\mathcal{I P} 2)$ such that

$$
\left\|B^{k+1} w(t)-w_{0}\right\|_{X} \rightarrow 0 \quad \text { as } t \rightarrow 0 .
$$

On the other hand, if $f \in \mathcal{C}^{k}([0, \tau] ; \mathbb{R})$ with $f^{(j)}(0)=0$ for $j=0,1, \ldots, k-1$, and $w_{0} \in \mathcal{R}\left(B^{k+1}\right)$, then $(\mathcal{I P} 2)$ possesses a unique solution $w$.

In accordance with what has been said, we arrive at the following assertions.

Theorem 2 Let $X$ be a Banach space; let $B \in \mathcal{L}(X)$ have $\lambda=0$ as the unique singularity of $(\lambda-B)^{-1}$ with a pole of order $k+1$; let $z \in X ;$ let $g \in \mathcal{C}^{1}([0, \tau] ; \mathbb{R})$ and let $\phi \in X \rightarrow \mathbb{R}$ be a $\mathcal{C}^{1}$ functional. Let $\phi[(I-P) z] \neq 0$, where $P$ is the projection onto $\mathcal{K}\left(B^{k+1}\right)$ along $\mathcal{R}\left(B^{k+1}\right)$. Then, for any $w_{0} \in \mathcal{R}\left(B^{k+1}\right)$, the identification problem $(\mathcal{I P} 2)$ possesses a unique solution $(w, f)$ such that

$$
w \in \mathcal{C}^{1}([0, \tau] ; X), \quad f \in \mathcal{C}^{k}([0, \tau] ; \mathbb{R}) .
$$

Theorem 3 Let $X$ be a Banach space; let $B$ have $\lambda=0$ as a pole of $(\lambda-B)^{-1}$ of order $k+1$; let $z \in X ;$ let $g \in \mathcal{C}^{1}([0, \tau] ; \mathbb{R})$ and let $\phi \in X \rightarrow \mathbb{R}$ be a $\mathcal{C}^{1}$ functional. Let $\phi[(I-P) z] \neq 0$, where $P$ is the projection onto $\mathcal{K}\left(B^{k+1}\right)$ along $\mathcal{R}\left(B^{k+1}\right)$. Finally, let us assume the initial value $w_{0}$ of the form (25) and $w_{0} \in \mathcal{R}\left(B^{k+1}\right)$. Then there exists a unique strict solution $(w, f)$ to the identification problem $(\mathcal{I P} 2)$ such that

$$
w \in C^{1}([0, \tau] ; X), \quad f \in \mathcal{C}^{k}([0, \tau] ; \mathbb{R}) .
$$

We are now ready to pass to the statement of the main result.

Theorem 4 Let $M$ and $L$ be two closed linear operators in a Banach space $X$ with $D(L) \subset$ $D(M)$, L being invertible, with the property that $\lambda=0$ is a pole of order $k+1$ of the operator $(\lambda-B)^{-1}$, where $B=M L^{-1}$. Let $z, v_{0} \in X, g \in \mathcal{C}^{1}([0, \tau] ; \mathbb{R})$ and $\phi \in X^{*}, X^{*}$ being the dual space to $X$. If in addition, $\phi[(I-P) z] \neq 0$, where $P$ is the projection onto $\mathcal{K}\left(B^{k+1}\right)$ along $\mathcal{R}\left(B^{k+1}\right)$, then there exists a unique strict solution $(v, f)$ to the identification problem $(\mathcal{I} \mathcal{P} 1)$ admitting the implicit representation

$$
\begin{aligned}
f(t)= & \gamma(z)\left(g^{\prime}(t)-\phi\left[B_{1}^{-1} S(t) u_{0}\right]\right)-\gamma(z) \phi\left[\int_{0}^{t} B_{1}^{-1} S(t-s) g^{\prime}(s)(I-P) z d s\right] \\
\nu(t)= & \sum_{j=0}^{k} L^{-1} B_{2}^{j} P f^{(j)}(t) z+L^{-1} B_{1}^{-1} S(t)(I-P) M v_{0} \\
& +\gamma(z) L^{-1}\left[\int_{0}^{t} B_{1}^{-1} S(t-s) g^{\prime}(s)(I-P) z d s\right]
\end{aligned}
$$


where $B_{1}$ and $B_{2}$ denote the parts of $B$ in $\mathcal{R}\left(B^{k+1}\right)$ and in $\mathcal{K}\left(B^{k+1}\right)$, respectively.

Proof Consider the identification problem $(\mathcal{I P} 1)$ where $M$ and $L$ are two closed linear operators in $X$ with $\mathcal{D}(L) \subset \mathcal{D}(M), L$ being invertible, with the property that $\lambda=0$ is a pole of order $k+1, k=0,1,2, \ldots$, of the bounded operator $L(\lambda L-M)^{-1}, z, v_{0} \in X, g \in \mathcal{C}^{1}([0, \tau] ; \mathbb{R})$ and $\phi \in X^{*}$.

We observe that $B=M L^{-1} \in \mathcal{L}(X)$ and

$$
(\lambda-B)^{-1}=L(\lambda L-M)^{-1}
$$

Let $w=L v$, then $(\mathcal{I P} 1)$ can be rewritten as $(\mathcal{I P} 2)$ where $w_{0}=M v_{0}$. A direct application of Theorem 2 implies the required existence and uniqueness result. The function $f(t)$ is determined using (21). Moreover, we have the explicit solution $v(t)$ from (24) as follows:

$$
\begin{aligned}
v(t)= & L^{-1} w(t) \\
= & \sum_{j=0}^{k} L^{-1} B_{2}^{j} P f^{(j)}(t) z+L^{-1} B_{1}^{-1} S(t)(I-P) M v_{0} \\
& +\gamma(z) L^{-1}\left[\int_{0}^{t} B_{1}^{-1} S(t-s) g^{\prime}(s)(I-P) z d s\right],
\end{aligned}
$$

and the proof is completed.

\section{Differential equations of Sobolev type}

Sobolev-type equations appear in a variety of physical problems such as flow of fluid through fissured rocks, thermodynamics, heat conduction involving two temperatures and soil mechanics. There is an extensive literature in which Sobolev-type equations are investigated in the abstract framework; see, for instance, [30-33]. As far as we know, the present paper is the first one to consider identification problems for differential equations of Sobolev type.

Let $K$ be a densely defined closed linear operator in a Banach space $X$. Assume that -1 is an eigenvalue of $K$ of multiplicity one, that is, $\lambda=0$ is a simple pole for the resolvent $(\lambda-1-K)^{-1}$. Then there exists $\epsilon>0$ such that

$$
\left\|(\lambda-1-K)^{-1}\right\|_{\mathcal{L}(X)} \leq \frac{C}{|\lambda|}, \quad 0<\lambda \leq \epsilon .
$$

We are concerned with the Sobolev-type differential equation

$$
\begin{aligned}
& \frac{d}{d t}(1+K) v=K v+f(t) z, \quad 0<t \leq T \\
& (1+K) v(0)=(1+K) v_{0}
\end{aligned}
$$

with the subsidiary information

$$
\phi[(1+K) v(t)]=g(t), \quad 0 \leq t \leq T,
$$


where $g(t)$ is a given function, $v_{0} \in \mathcal{D}(K)$ is an initial value, and where $v=v(t)$ and $f(t)$ are the unknown functions. By the change of the unknown function $\bar{v}(t)=e^{-(\mu+1) t} v(t)$ with some exponent $\mu$ to be specified below, our problem is transformed into

$$
\begin{aligned}
& \frac{d}{d t}(1+K) \bar{v}=-\mu(1+K) \bar{v}-\bar{v}+f_{\mu}(t) z, \quad 0<t \leq T, \\
& (1+K) \bar{v}(0)=(1+K) v_{0},
\end{aligned}
$$

where $f_{\mu}(t)=e^{-(\mu+1) t} f(t)$. So that this problem is viewed as a problem of the form $(\mathcal{I P} 1)$ with $M=1+K$ and $L=-\mu(1+K)-1$. Since

$$
\lambda M-L=(\lambda+\mu)\left(\frac{1}{\lambda+\mu}+M\right)
$$

the $M$ resolvent exists if $|1 /(\lambda+\mu)| \leq \epsilon$ or if $|\lambda+\mu| \geq 1 / \epsilon$. In addition, since

$$
M(\lambda M-L)^{-1}=\frac{1}{\lambda+\mu}\left\{1-\frac{1}{\lambda+\mu}\left(\frac{1}{\lambda+\mu}+M\right)^{-1}\right\}
$$

then $(\lambda M-L)^{-1}$ is a bounded operator on $X$ for all $|\lambda| \geq \epsilon_{0}>1 /(\lambda+\mu)$. Hence, for all $v$, $0<v<1 / \epsilon_{0}$ with a suitable $\epsilon_{0}>0$,

$$
(v L-M)^{-1}=v^{-1}\left(L-v^{-1} M\right)^{-1},
$$

and it is readily seen that $v=0$ is a simple pole for $M(v L-M)^{-1}$.

As an example of an identification problem for a Sobolev-type differential equation, we give

$$
\begin{aligned}
& \frac{\partial}{\partial t}\left(1+\frac{\partial^{2}}{\partial x^{2}}\right) v(t, x)=\frac{\partial^{2} v(x, t)}{\partial x^{2}}+f(t) z(x), \quad 0<t \leq T, 0 \leq x \leq \ell \pi \\
& v(0, t)=v(\ell \pi, t)=0, \quad 0<t \leq T, \\
& \left(1+\frac{\partial^{2}}{\partial x^{2}}\right) v(x, 0)=\left(1+\frac{\partial^{2}}{\partial x^{2}}\right) v_{0}(x), \quad 0 \leq x \leq \ell \pi
\end{aligned}
$$

subject to the additional information

$$
\int_{0}^{\ell \pi} w(x)\left(1+\frac{\partial^{2}}{\partial x^{2}}\right) v(t, x) d x=g(t), \quad 0 \leq t \leq T
$$

where $\ell$ is a positive integer, $w \in \mathcal{C}([0, \ell \pi] ; \mathbb{C}), z(\cdot) \in \mathcal{C}([0, \ell \pi] ; \mathbb{C}), v_{0} \in \mathcal{D}(K)$. Here $X$ is the space of continuous functions

$$
X=\{f \in \mathcal{C}([0, \ell \pi] ; \mathbb{C}): f(0)=f(\ell \pi)=0\}
$$

and the operator $K$ is given by

$$
\left\{\begin{array}{l}
\mathcal{D}(K)=\left\{v \in \mathcal{C}^{2}([0, \ell \pi] ; \mathbb{C}): v(0)=v(\ell \pi)=v^{\prime \prime}(0)=v^{\prime \prime}(\ell \pi)=0\right\}, \\
K v=\frac{d^{2} v}{d x^{2}}
\end{array}\right.
$$




\section{Applications}

We finally consider the abstract results as a source of existence and uniqueness theorems for the identification problems related to some degenerate systems.

Example 5 Let $L$ be a bounded operator in $\mathcal{L}(X)$ and $M$ be a densely defined closed operator in $X$ such that $\left(\lambda_{0} M-L\right)^{-1}$ is a compact operator in $X$. Since

$$
\lambda_{0} M\left(\lambda_{0} M-L\right)^{-1}=1+L\left(\lambda_{0} M-L\right)^{-1},
$$

we deduce that $S=\lambda_{0} M\left(\lambda_{0} M-L\right)^{-1}$ is a Fredholm operator with

$$
r(S)<\infty, \quad r^{*}(S)<\infty .
$$

Moreover, $\mathcal{R}(S)=\mathcal{R}(M)$ is closed in $X$ and this implies [34, pp.150-152] that $\lambda=0$ is a pole of $(\lambda-S)^{-1}$ with $X=\mathcal{K}\left(S^{m}\right) \oplus \mathcal{R}\left(S^{m}\right)$ for a certain positive integer $m$. Therefore Theorem 4 applies.

Example 6 Let $B$ be a closed linear operator with a compact resolvent. If $0 \in \sigma(B)$, then it is an isolated eigenvalue with finite multiplicity [28, p.181], and therefore Theorem 4 applies again.

Example 7 Let $B$ be a closed Fredholm operator in $X$ such that

$$
r(B)=\lim _{n \rightarrow \infty} \alpha\left(B^{n}\right)<\infty, \quad r^{*}(B)=\lim _{n \rightarrow \infty} \beta\left(B^{n}\right)<\infty,
$$

where, for any closed linear operator $S$ in $X, \alpha(S)$ denotes the dimension of $\mathcal{K}(S)$ and $\beta(S)$ is the codimension of $\mathcal{R}(S)$ in $X$. Then there is a positive integer $m$ such that $X=\mathcal{K}\left(S^{m}\right) \oplus$ $\mathcal{R}\left(S^{m}\right)\left[35\right.$, pp.274-275] and $\lambda=0$ is an isolated singularity of $(\lambda-B)^{-1}$.

Example 8 If $X$ is a Hilbert space and $B_{0}$ is a compact linear operator from $X$ into itself such that $\lambda_{0} \neq 0$ is an element of $\sigma\left(B_{0}\right)$, then Theorem 4 applies with $B=\lambda_{0}-B_{0}$.

Competing interests

The authors declare that they have no competing interests.

Authors' contributions

All authors contributed equally and significantly in this paper. All authors read and approved the final manuscript.

\section{Author details}

'Department of Mathematics, Hashemite University, Zarqa, 13115, Jordan. ${ }^{2}$ Mathematics and Sciences Department,

Dhofar University, Salalah, 211, Oman. ${ }^{3}$ Department of Mathematics, Yarmouk University, Irbid, 211-63, Jordan.

\section{Publisher's Note}

Springer Nature remains neutral with regard to jurisdictional claims in published maps and institutional affiliations.

Received: 13 July 2017 Accepted: 13 October 2017 Published online: 26 October 2017

\section{References}

1. Lorenzi, A, Vrabie, I: Identification for a semilinear evolution equation in a Banach space. Inverse Probl. 26, 1-16 (2010)

2. Johansson, BT, Lesnic, D: A variational method for identifying a spacewise-dependent heat source. IMA J. Appl. Math. 72, 748-760 (2007)

3. El Badia, A, Ha-Duong, T: On an inverse source problem for the heat equation. Application to a pollution detection problem. J. Inverse III-Posed Probl. 10, 585-599 (2002) 
4. Cannon, JR: Determination of an unknown heat source from overspecified boundary data. SIAM J. Numer. Anal. 5, 275-286 (1968)

5. Farcas, A, Lesnic, D: The boundary-element method for the determination of a heat source dependent on one variable. J. Eng. Math. 54, 375-388 (2005)

6. Engl, HW, Scherzer, O, Yamamoto, M: Uniqueness and stable determination of forcing terms in linear partial differential equations with overspecified boundary data. Inverse Probl. 10, 1253-1276 (1994)

7. Kusiak, S, Weatherwax, J: Identification and characterization of a mobile source in a general parabolic differential equation with constant coefficients. SIAM J. Appl. Math. 68, 784-805 (2008)

8. Prilepko, I, Orlovsky, G, Vasin, A: Methods for Solving Inverse Problems in Mathematical Physics. Dekker, New York (2000)

9. Orlovsky, D: An inverse problem for a second order differential equation in a Banach space. Differ. Equ. 25, 1000-1009 (1989)

10. Orlovsky, D: Weak and strong solutions of inverse problems for differential equations in a Banach space. Differ. Equ. 27, 867-874 (1991)

11. Lorenzi, A: An Introduction to Identification Problems, Via Functional Analysis. VSP, Utrecht (2001)

12. Awawdeh, F: Perturbation method for abstract second-order inverse problems. Nonlinear Anal. 72, 1379-1386 (2010)

13. Al Horani, M: An identification problem for some degenerate differential equation. Matematiche 57(1), 217-227 (2002)

14. Al Horani, M, Favini, A: An identification problem for first-order degenerate differential equations. J. Optim. Theory Appl. 130(1), 41-60 (2006)

15. Al Horani, M: Projection method for solving degenerate first-order identification problem. J. Math. Anal. Appl. 364(1), 204-208 (2010)

16. Al Horani, M, Favini, M, Lorenzi, A: Second-order degenerate identification differential problems. J. Optim. Theory Appl. 141(1), 13-36 (2009)

17. Favini, A, Yagi, A: Degenerate Differential Equations in Banach Spaces. Dekker, New York (1999)

18. Awawdeh, F, Obiedat, HM: Source identification problem for degenerate differential equations. UPB Sci. Bull., Ser. A 73(1), 61-72 (2011)

19. Fedorov, VE, Ivanova, ND: Identification problem for a degenerate evolution equation with overdetermination on the solution semigroup kernel. Discrete Contin. Dyn. Syst., Ser. S 9(3), 687-696 (2016)

20. Favini, A, Lorenzi, A: Identification problems for singular integro-differential equations of parabolic type II. Nonlinear Anal. 56, 879-904 (2004)

21. Fedorov, VE, Ivanova, ND: Identification problem for degenerate evolution equations of fractional order. Fract. Calc. Appl. Anal. 20, 706-721 (2017)

22. Kostić, M: Abstract degenerate fractional differential inclusions in Banach spaces. Appl. Anal. Discrete Math. 11, 39-61 (2017)

23. Carroll, RW, Showalter, RE: Singular and Degenerate Cauchy Problems. Academic Press, New York (1976)

24. Kostić, M: Abstract Degenerate Volterra Integro-Differential Equations. Book manuscript (2016)

25. Sviridyuk, GA, Fedorov, VE: Linear Sobolev Type Equations and Degenerate Semi-Groups of Operators. VSP, Utrecht (2003)

26. Favini, A, Marinoschi, G: Identification for degenerate problems of hyperbolic type. Appl. Anal. 91(8), 1511-1527 (2012)

27. Yoshida, K: Functional Analysis, 6th edn. Springer, Berlin (1980)

28. Kato, T: Perturbation Theory of Linear Operators. Springer, Berlin (1966)

29. Desch, W, Schappacher, W: On relatively bounded perturbations of linear $c_{0}$-semigroups. Ann. Sc. Norm. Super. Pisa, Cl. Sci. 11(2), 327-341 (1984)

30. Krishnan, B, Subbarayan, K: Regularity of solutions of Sobolev-type semilinear integrodifferential equations in Banach spaces. Electron. J. Differ. Equ. 2003, 114 (2003)

31. Lightbourne, JH, Rankin, SM: A partial functional differential equation of Sobolev type. J. Math. Anal. Appl. 93, 328-337 (1983)

32. Showalter, RE: A nonlinear parabolic Sobolev equation. J. Math. Anal. Appl. 50, 183-190 (1975)

33. Sobolev, S: Some new problems in mathematical physics. Izv. Akad. Nauk SSSR, Ser. Mat. 18, 3-50 (1954)

34. Schechter, M: Principles of Functional Analysis. Academic Press, New York (1971)

35. Schechter, M: Basic theory of Fredholm operators. Ann. Sc. Norm. Super. Pisa 21, 361-380 (1967)

\section{Submit your manuscript to a SpringerOpen ${ }^{\circ}$ journal and benefit from:}

- Convenient online submission

- Rigorous peer review

- Open access: articles freely available online

- High visibility within the field

- Retaining the copyright to your article

Submit your next manuscript at $\gg$ springeropen.com 